\title{
Effect of estrogen and progesterone on nerve conduction studies during ovarian cycle
}

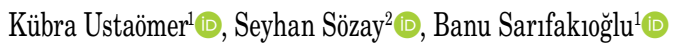 \\ ${ }^{1}$ Department of Physical Medicine and Rehabilitation, Tekirdağ Namık Kemal University Faculty of Medicine, Tekirdağ, Turkey \\ ${ }^{2}$ Department of Physical Medicine and Rehabilitation, Başkent University Faculty of Medicine, Ankara, Turkey
}

Received: May 15, 2020 Accepted: October 13, 2020 Published online: December 01, 2021

\begin{abstract}
Objectives: This study aims to investigate the effects of estrogen and progesterone on nerve conduction studies (NCSs) in three different hormonal phases of the ovarian cycle.

Patients and methods: Between April 2008 and July 2008, a total of 40 healthy volunteer women (mean age: $24.1 \pm 5.1$ years; range 21 to 43 years) with regular menstrual cycles were included in this prospective study. The participants were regularly menstruating for at least one year, without any hormonal disease and without taking any medication that could lead to hormonal dysregulation. Motor and sensory conduction velocities, amplitudes, and distal latencies were analyzed at the dominant extremities within the early follicular phase (EFP), late follicular phase (LFP), and the midluteal phase (MLP).

Results: Except for the median nerve motor conduction velocity (MCV), there were no statistically significant differences between the peripheral NCS results in the three ovarian cycle phases $(\mathrm{p}=0.033)$. After adjusting for multiple comparisons, a significant difference was found between the EFP and LFP ( $\mathrm{p}=0.004)$.

Conclusion: Our study results showed that only median nerve MCV was affected in the menstrual cycle. However, this would be an incidental finding, or an increased sensibility of the median nerve motor fibers to ovarian steroids by an unknown mechanism. Further studies are warranted.
\end{abstract}

Keywords: Electromyography, median nerve, nerve conduction, ovarian cycle.

Nerve conduction studies (NCSs) are affected by several physiological factors, such as age, height, and temperature. ${ }^{[1]}$ Over the last three decades, many researchers have studied neuroactive steroids (NASs) and their effects on nerve physiology. The NAS is a general term given to all steroids that affect the function of the nervous system. ${ }^{[2]}$ These agents can be synthesized in the central nervous system (CNS), peripheral nervous system (PNS), and peripheral glands, such as the ovaries and adrenal glands. ${ }^{[2]}$ Several reviews have shown that CNS and PNS are able to produce neurosteroids, and they are also targets for NAS. ${ }^{[2-6]}$
Estrogen and progesterone are ovarian hormones involved in neuroendocrine control of reproduction. ${ }^{[7]}$ They are also involved in the physiological regulation of neural functions: neurogenesis, neuronal survival, neuronal differentiation, synaptogenesis, glial differentiation, myelin formation, synaptic function, and synaptic plasticity. In the presence of pathological conditions, they exert neuroprotective actions, promoting neuronal survival and remyelination and decreasing neuroinflammation. ${ }^{[3]}$

Electrophysiological studies are the main procedures that are used to evaluate peripheral

Corresponding author: Kübra Ustaömer, MD. Namık Kemal Üniversitesi Tıp Fakültesi, Fiziksel Tıp ve Rehabilitasyon Anabilim Dalı, 59030 Tekirdağ, Türkiye. e-mail: qustaomer@hotmail.com 
nerve functions. However, the effects of estrogen and progesterone on NCS during an ovarian cycle have not been clearly known, yet. In the present study, we aimed to investigate the effects of estrogen and progesterone on electrophysiological parameters, such as peripheral NCS and late responses (LRs) within the early follicular phase (EFP), late follicular phase (LFP), and midluteal phase (MLP) of the ovarian cycle.

\section{PATIENTS AND METHODS}

This single-center, prospective clinical study was conducted at Başkent University Faculty of Medicine, Department of Physical Medicine and Rehabilitation, Electroneuromyography Unit between April 2008 and July 2008. A total of 40 healthy volunteer women (mean age: $24.1 \pm 5.1$ years; range 21 to 43 years) with regular menstrual cycles were included in the study. All participants had regularly menstruated for at least for one year, without any hormonal disease and without taking any medication that could lead to hormonal dysregulation. The duration of the menstrual cycle was 28 days. Exclusion criteria were as follows: having a history of disease or medication affecting the ovarian cycle (e.g., polycystic ovary syndrome, thyroid disease, oral contraceptive, or non-steroidal anti-inflammatory drugs), and clinical conditions resulting in peripheral neuropathy (e.g., vitamin B12 deficiency, steroid usage, or diabetes). The study flow chart is shown in Figure 1. A written informed consent was obtained from each participant. The study protocol was approved by the Başkent University Faculty of Medicine Clinical Research Ethics Committee (No: KA08/30). The study was conducted in accordance with the principles of the Declaration of Helsinki.

\section{Electrophysiological studies}

Electrophysiological studies were conducted in accordance with the protocol recommended by the American Association of Electrodiagnostic Medicine (AAEM) using the Medelec ${ }^{\circledR}$ Synergy Multimedia EMG/EP (Oxford Instruments, Surrey, UK) by a single physician who was blinded to the ovarian cycle phase of the participants. ${ }^{[8-14]}$

In an average cycle lasting for 28 days, the studies were performed on Days 3, 13, and 23 of the cycle. Data on baseline body temperatures were obtained daily, followed by the participants confirming that they were ovulating and in the luteal phase (LP). The first electrophysiological study was done in the EFP, when the estrogen and progesterone levels were low. The second study was done in the LFP approximately
24 to $48 \mathrm{~h}$ before ovulation with the highest estrogen and low progesterone levels of the cycle. The third study was done 10 days after the second study and five days before the expected menstruation in the MLP, when the progesterone levels were the highest and the estrogen levels were moderately lower than the other phases.

The electrophysiological studies consisted of median, ulnar, tibial, and peroneal nerve motor conduction studies (MCSs), median, ulnar, radial, and sural nerve sensory conduction studies (SCSs) and LRs. ${ }^{[1,8-14]}$ Compound muscle action potential (CMAP) amplitudes, sensorial nerve action potential (SNAP) amplitudes, motor and sensorial nerve conduction velocities, distal motor and distal sensory latencies, and LR (F latency of the motor nerve, $\mathrm{H}$ reflex of the soleus muscle) were recorded at the dominant side of the body. ${ }^{[1,8-14]}$

Electrophysiological studies were performed early in the morning by controlling the skin temperature ( 32 to $34^{\circ} \mathrm{C}$ ) at a standard room temperature. All the electrophysiological studies were conducted using the same device and by the same practitioner blinded to the phase of the ovarian cycle of each participant.

\section{Statistical analysis}

Statistical analysis was performed using the SPSS for Windows version 15.0 software (SPSS Inc., Chicago, IL, USA). Descriptive data were presented in mean \pm standard deviation (SD), median (min-max) or number and frequency, where applicable. The normality of the data was checked using the ShapiroWilk test. For repetitive measures, the presence of a statistically significant difference between the measurements was analyzed using variance analysis of repeated measures. The Bonferroni correction for multiple comparisons test was used to identify which measurement caused the difference. A $p$ value of $<0.05$ was considered statistically significant.

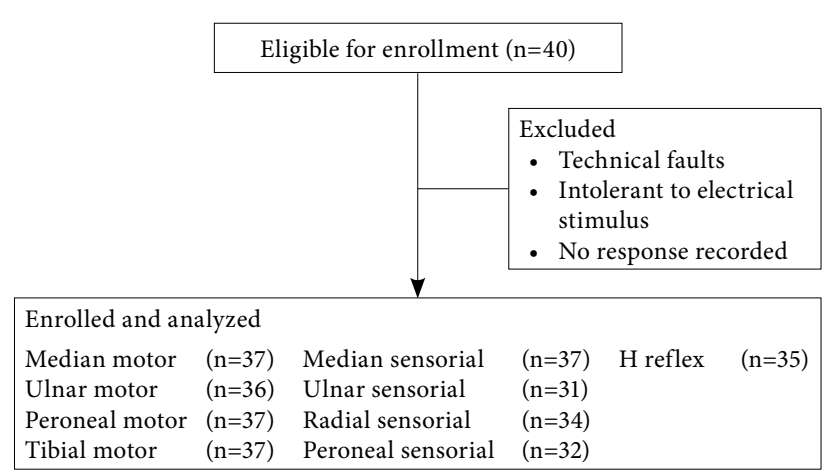

Figure 1. Study flow chart. 


\section{RESULTS}

Baseline demographic characteristics of the participants are summarized in Table 1. Incomplete data due to anatomic variations, technical faults, and pain-related unavailable responses were excluded in all three phases. The remaining data were statistically analyzed. The phases and hormonal status of an ovarian cycle are shown in Figure 2.

The motor and sensory NCS results for each ovarian phase are summarized in Table 2 and Table 3, respectively. A significant difference in the median nerve MCV was found between the three phases of the ovarian cycle $(\mathrm{p}=0.033)$ (Table 2$)$. After adjusting for multiple comparisons (Bonferroni), significant differences were found between the EFP and LFP $(\mathrm{p}=0.004)$; however, no significant difference was observed between the EFP and MLP $(\mathrm{p}=1.000)$ and between LFP and MLP ( $\mathrm{p}=0.239)$.

There were no significant differences in the MCV of the ulnar, tibial, and peroneal nerves (Table 2) and the SCV of the median, ulnar, radial, peroneal, and sural nerves (Table 3). Moreover, no amplitude and latency changes were observed (Table 4).

\begin{tabular}{|lc|}
\hline \multicolumn{2}{|c|}{ TABLE 1 } \\
\hline \multicolumn{2}{|c|}{ Demographic data of participants } \\
\hline Age (year) & Mean \pm SD \\
Height $(\mathrm{cm})$ & $29.1 \pm 5.1$ \\
Body weight $(\mathrm{kg})$ & $161 \pm 10.9$ \\
Body mass index $\left(\mathrm{kg} / \mathrm{m}^{2}\right)$ & $61.9 \pm 10.9$ \\
\hline SD: Standard deviation. & $23.5 \pm 4.5$ \\
\hline
\end{tabular}

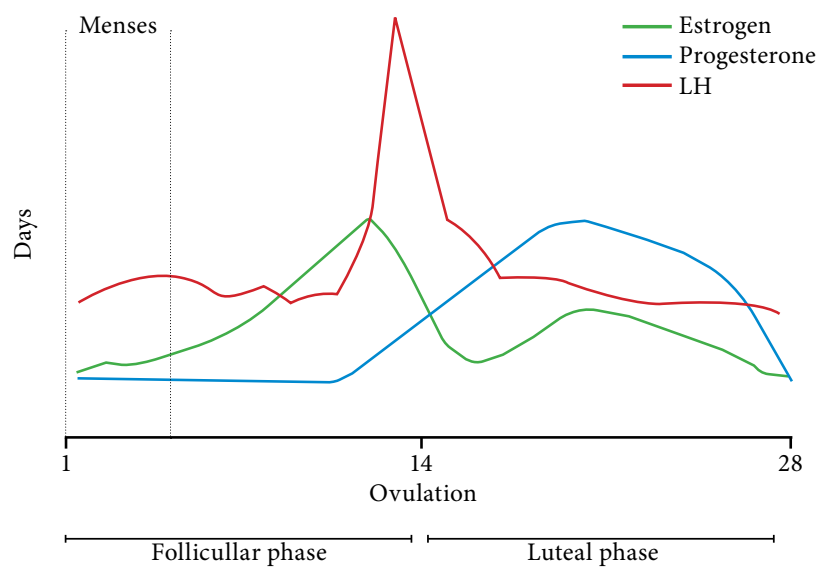

Figure 2. Hormonal status of an ovarian cycle.

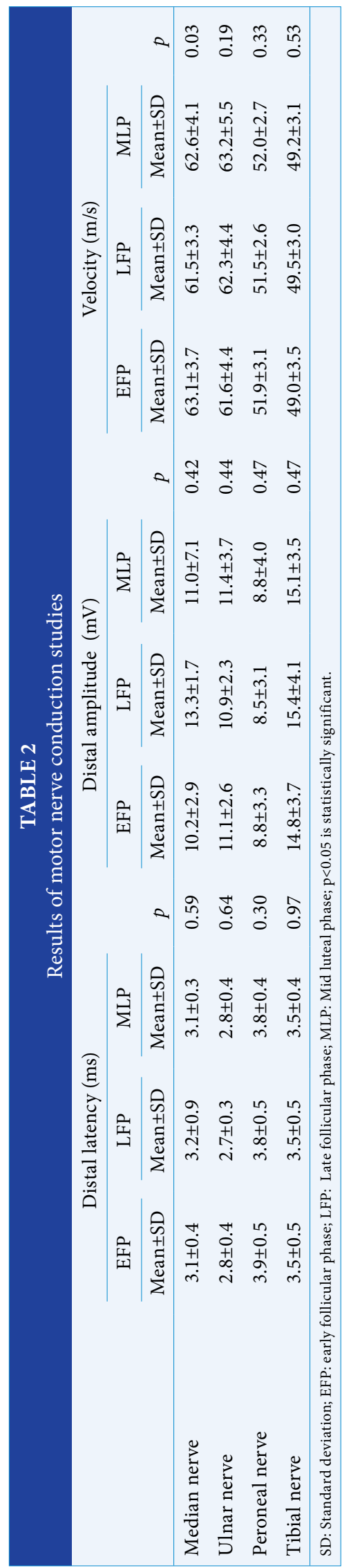




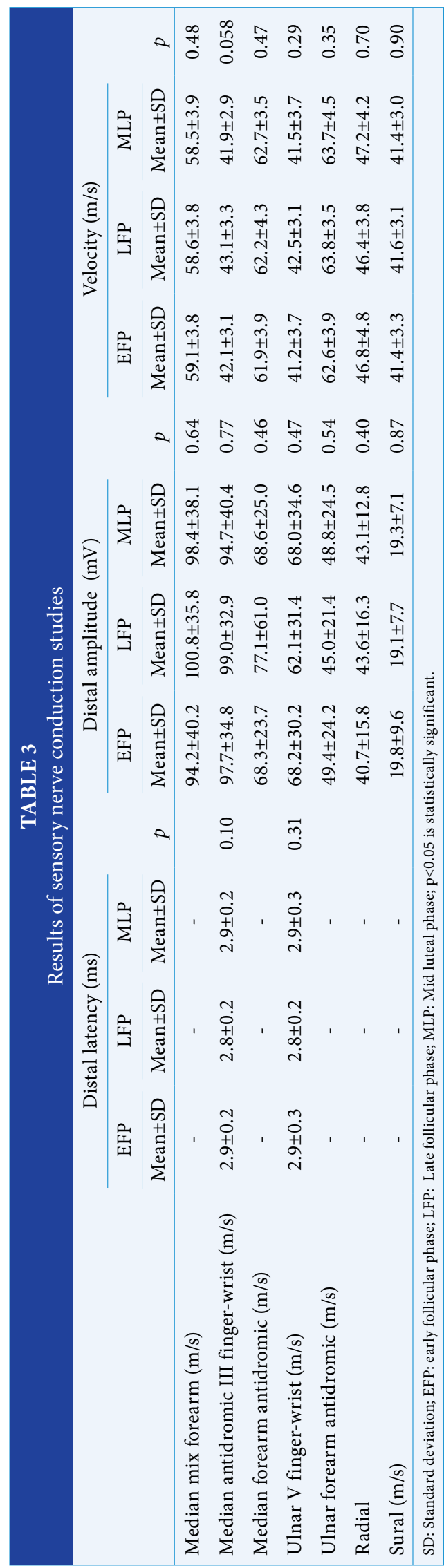

\begin{tabular}{|c|c|c|c|c|}
\hline \multicolumn{5}{|c|}{$\begin{array}{c}\text { TABLE } 4 \\
\text { Electrophysiological findings in late responses of studies } \\
\text { of participants }\end{array}$} \\
\hline & EFP & LFP & MLP & \\
\hline & Mean \pm SD & Mean \pm SD & Mean \pm SD & $p$ \\
\hline Median F-min (ms) & $23.2 \pm 1.2$ & $23.4 \pm 1.6$ & $23.5 \pm 1.3$ & 0.20 \\
\hline Ulnar F-min (ms) & $24.5 \pm 6.1$ & $23.4 \pm 1.4$ & $23.3 \pm 1.3$ & 0.28 \\
\hline Peroneal F-min (ms) & $42.8 \pm 3.0$ & $44.3 \pm 3.4$ & $42.7 \pm 2.7$ & 0.66 \\
\hline Tibial F-min (ms) & $43.8 \pm 3.3$ & $44.3 \pm 3.4$ & $43.7 \pm 3.2$ & 0.70 \\
\hline H reflex (ms) & $23.3 \pm 9.1$ & $24.0 \pm 8.0$ & $23.5 \pm 9.9$ & 0.26 \\
\hline
\end{tabular}

\section{DISCUSSION}

Many electrophysiological studies have investigated the effects of steroidal hormones on the physiology of the central and peripheral nerves. The electrophysiological parameters that are most often studied during the normal hormonal cycle in healthy women are latency changes of the visual evoked potentials (VEPs) and the brainstem auditory evoked potentials (BAEPs). These potentials provide information about the peripheral and central components of the nervous system. To the best of our knowledge, no previous study in the literature has investigated the hormonal effects of the ovarian cycle on peripheral nerve conduction so far. In our study, nerve conduction was recorded in normal healthy women with regular menstrual cycles. We found that median motor NCV was affected during EFP, when both the estrogen and progesterone levels were low.

In a study including 10 participants conducted by Soares et al., ${ }^{[15]}$ a decrease in the conduction velocity during menstruation and immediately after ovulation was reported; however, these results did not reach statistical significance. In addition, this study did not perform routine NCS, but only recorded surface electromyographic signals from the biceps brachii muscle. In our study, on the contrary, we performed routine upper and lower extremity NCSs.

In various studies, changes in VEP latency and amplitudes were seen in different phases of the ovarian cycle. ${ }^{[16-18]}$ Azarmina et al. ${ }^{[19]}$ reported a prolongation of VEP latency in the LP. They interpreted their result as the neuroinhibitory effect of progesterone on optic nerve conduction. In another study, Yilmaz et al. ${ }^{[20]}$ recorded shorter latencies during the ovulatory phase. They noted that their results were due to the effect of estrogen on the neural transmission of the visual 
pathways. In these studies, the results are explained by the neuroinhibitory effects of progesterone or the neuroexcitatory effects of estrogen. In the literature, BAEP studies have reported alterations in the LP. ${ }^{[21,22]}$ Elkind-Hirsch et al. ${ }^{[23]}$ showed that the BAEP changes were due to the elongation in the transmission time caused by estrogen and the antagonizing effect of progesterone. Batta et al. ${ }^{[24]}$ concluded that, in the secretory phase, the thermogenic effects of progesterone led to an increase in neural conduction. The diverse results reported in VEP and BAEP studies may be due to differences in the NAS effects in various parts of the CNS. Unlike these studies, the latency differences were not observed in our study. This difference may be related to differences in the NAS levels in the CNS and PNS. Moreover, the effects of NAS may differ depending on the type of nervous system tissue.

In our study, for the first time in the literature, peripheral NCV was studied in the dominant upper and lower extremities during three phases of the ovarian cycle in women with regular menstrual cycles. We found that the median motor NCV was significantly different between the EFP and LFP. Both estrogen and progesterone were in their baseline levels in EFP. Thus, the increment of median motor NCV in EFP cannot be explained by myelinization, neuroexcitatory, or neuroprotective effects of estrogen and progesterone. One of the possible explanations for the differences in the velocity between EFP and LFP is the neuroinhibitory effect of the increasing levels of estrogen in LFP. Thereafter, a non-significant increase in NCV in MLP suggested that the progesterone had an antagonizing effect on the inhibitory effect of estrogen, similar to what was reported in the study by Elkind-Hirsch et al. ${ }^{[23]}$ The aldosterone affinity to $G$ protein-coupled estrogen receptor 1 (GPER) and the interaction between steroid hormones and mineralocorticoid receptors (MRs) are the other mechanisms that may explain the NCV differences between EFP and LFP. ${ }^{[25-29]}$ The MR retains significant similarities to progesterone receptor (PR). ${ }^{[26]}$ Progesterone is a competitive MR antagonist. ${ }^{[27]}$ In the LP, the inhibition of MR by progesterone doubles the aldosterone levels. ${ }^{[27]}$ This may explain the complaints that many women suffer from body edema during the LP stage of their ovarian cycle; edema is alleviated at the end of the LP due hormonal withdrawal. ${ }^{[30,31]}$ In the follicular phase (FP) and LP, increasing estrogen and progesterone levels may lead to water and salt retention by MR and a fluid shift from the intravascular space to the extracellular space. ${ }^{[7,30]}$ Edema and pressure around the median nerve may result in a decrease in the median MCV. ${ }^{[32]}$ At the end of LP, there is a sudden drop in the estrogen and progesterone levels. Subsequently, menstrual diuresis occurs in the EFP. ${ }^{[30]}$ The mineralocorticoid effects of ovarian steroids and related edema and pressure resolve which may explain the increase in the median MCV in EFP.

Based on our findings, only the median nerve MCV was affected. The difference in the median nerve NCS may depend on the narrow structure of the carpal tunnel in women. ${ }^{[33]}$ Furthermore, there is a tendency for the median nerve to be entrapped in the carpal tunnel in women. ${ }^{[34]}$ All these factors (narrow structure, tendency toward entrapment, mineralocorticoid effects of hormones) may be related to the effect on the median motor NCS. No differences were observed in the other peripheral NCS, including median nerve sensory responses. This could be an incidental finding. On the other hand, a probable sensitivity of the median nerve recurrent motor fibers to hormonal effects should be considered.

The present study has certain limitations. First, it has a small number of participants. However, our findings can be supported by the results of future large scale studies. Another limitation is the lack of confirmation of the phases with blood samples; the samples were unable to be obtained due to economic issues. Even so, we attempted to confirm three phases of the ovarian cycle by including women with regular menstrual cycles and by requesting that the participants to check their baseline body temperature daily to establish their ovulation time. Also, a group of young women in surgically-induced menopause could be added to study as a control group. Finally, room temperature and technical factors such as stimulus artifact, electrode placement for motor studies, distance between recording electrodes and nerve, distance between active and reference recording electrodes, limb position and distance measurements can affect nerve conduction, although we attempted to minimize all of these factors.

In conclusion, although it is well known that physiological factors have an effect on electrophysiological studies, the exact effects of ovarian hormones on NCV are still unclear. In our study, we found that faster median motor NCV in the EFP. This result can be interpreted as a more selectively affected recurrent motor branch of median nerve by estrogen and progesterone or as an incidental finding. Nevertheless, further large-scale studies are needed to confirm the hormonal status of each cycle phase using 
imaging methods such as ultrasonography or magnetic resonance imaging.

\section{Declaration of conflicting interests}

The authors declared no conflicts of interest with respect to the authorship and/or publication of this article.

\section{Funding}

The authors received no financial support for the research and/or authorship of this article.

\section{REFERENCES}

1. Preston DC, Shapiro BE. Sources of Error: Anomalies, Artifacts, Technical Faults, and Statistics: In: Preston DC, Shapiro BE, editors. Electromyography and neuromuscular disorders: Clinical-electrophysiologic correlations. 3rd ed. China: Elsevier Saunders; 2013. p. 62-96.

2. Melcangi RC, Garcia-Segura LM, Mensah-Nyagan AG. Neuroactive steroids: State of the art and new perspectives. Cell Mol Life Sci 2008;65:777-97.

3. Melcangi RC, Giatti S, Garcia-Segura LM. Levels and actions of neuroactive steroids in the nervous system under physiological and pathological conditions: Sex-specific features. Neurosci Biobehav Rev 2016;67:25-40.

4. Melcangi RC, Giatti S, Pesaresi M, Calabrese D, Mitro N, Caruso D, et al. Role of neuroactive steroids in the peripheral nervous system. Front Endocrinol (Lausanne) 2011;2:104.

5. Porcu P, Barron AM, Frye CA, Walf AA, Yang SY, He $X Y$, et al. Neurosteroidogenesis Today: Novel targets for neuroactive steroid synthesis and action and their relevance for translational research. J Neuroendocrinol 2016;28:12351.

6. Panzica GC, Balthazart J, Frye CA, Garcia-Segura LM, Herbison AE, Mensah-Nyagan AG, et al. Milestones on steroids and the nervous system: 10 years of basic and translational research. J Neuroendocrinol 2012;24:1-15.

7. Guyton AC, Hall JE, editors. Endocrinology and Reproduction: Female physiology before pregnancy and female organs: Guyton and Hall: Textbook of medical physiology. 13th ed. Philadelphia: Elsevier; 2016. p. 1037-54.

8. Guidelines in electrodiagnostic medicine. American Association of Electrodiagnostic Medicine. Muscle Nerve 1992;15:229-53.

9. Guidelines for ethical behavior relating to clinical practice issues in electrodiagnostic medicine. American Association of Electrodiagnostic Medicine. Muscle Nerve 1994;17:965-7.

10. Oh SJ. Nerve conduction tehniques. General concept of electrodiagnostic studies in neuromuscular diseases: Clinical electromyography, nerve conduction studies. 3rd ed. Philedelphia: Lippincott Williams \& Wilkins. Basic Section; 2003. p. 37-53.

11. Preston DC, Shapiro BE, editors. Fundementals of Nerve Conduction Studies: Electromyography and Neuromuscular Disorders: Clinical-Electrophysiologic Correlations. 3rd ed. China: Elsevier Saunders; 2013. p. 19-61.

12. Preston DC, Shapiro BE, editors. Detailed Nerve Conduction Studies: Electromyography and Neuromuscular Disorders:
Clinical-Electrophysiologic Correlations. 3rd ed. China: Elsevier Saunders; 2013. p. 97-124.

13. Fisher MA. AAEM Minimonograph \#13: $H$ reflexes and F waves: Physiology and clinical indications. Muscle Nerv 1992;15:1223-33.

14. Oh SJ. Anatomical guide for common nerve conduction studies: General concept of electrodiagnostic studies in neuromuscular diseases. Clinical electromyography, nerve conduction studies. 3rd ed. Philedelphia. Lippincott Williams \& Wilkins. Basic section 1.5. 2003. p. 54-78.

15. Soares FA, Salomoni SE, Veneziano WH, de Carvalho JL, Nascimento FA, Pires KF, et al. On the behavior of surface electromyographic variables during the menstrual cycle. Physiol Meas 2011;32:543-57.

16. Shushtarian SM, Yahyavi SH. Study of visual evoked potentials during normal monthly cycle in normal female subjects. Biomed Sci Instrum 1999;35:165-7.

17. Avitabile T, Longo A, Caruso S, Gagliano C, Amato $\mathrm{R}$, Scollo $\mathrm{D}$, et al. Changes in visual evoked potentials during the menstrual cycle in young women. Curr Eye Res 2007;32:999-1003.

18. Kaneda Y, Ikuta T, Nakayama H, Kagawa K, Furuta N. Visual evoked potential and electroencephalogram of healthy females during the menstrual cycle. J Med Invest 1997;44:41-6.

19. Azarmina M, Soheilian M, Azarmina H. Increased latency of visual evoked potentials in healthy women during menstruation. J Ophthalmic Vis Res 2011;6:183-6.

20. Yilmaz H, Erkin EF, Mavioğlu H, Sungurtekin U. Changes in pattern reversal evoked potentials during menstrual cycle. Int Ophthalmol 1998;22:27-30.

21. Mann N, Sidhu RS, Babbar R. Brainstem auditory evoked responses in different phases of menstrual cycle. J Clin Diagn Res 2012;6:1640-3.

22. Upadhayay N, Paudel BH, Singh PN, Bhattarai BK, Agrawal K. Pre- and postovulatory auditory brainstem response in normal women. Indian J Otolaryngol Head Neck Surg 2014;66(Suppl 1):133-7.

23. Elkind-Hirsch KE, Wallace E, Malinak LR, Jerger JJ. Sex hormones regulate ABR latency. Otolaryngol Head Neck Surg 1994;110:46-52.

24. Batta M, Dhir SK, Kumar A, Singh KD. Effect of different phases of menstrual cycle on brainstem auditory evoked response. Int J Appl Basic Med Res 2017;7:44-7.

25. Ding Q, Chorazyczewski J, Gros R, Feldman R. Aldosterone binding to G Protein Estrogen Receptor-GPER. FASEB J 2019:33:503.

26. Ong GS, Young MJ. Mineralocorticoid regulation of cell function: The role of rapid signalling and gene transcription pathways. J Mol Endocrinol 2017;58:R33-R57.

27. Gomez-Sanchez E, Gomez-Sanchez CE. The multifaceted mineralocorticoid receptor. Compr Physiol 2014;4:965-94.

28. Rossi GP, Caroccia B, Seccia TM. Role of estrogen receptors in modulating aldosterone biosynthesis and blood pressure. Steroids 2019;152:108486.

29. Wendler A, Albrecht C, Wehling M. Nongenomic actions of aldosterone and progesterone revisited. Steroids 2012;77:1002-6. 
30. Ryan WL, Luby RJ, Haller MJ. The relation of urine volume to the menstrual cycle and ovulation. Fertil Steril 1961;12:589-94.

31. Thorn GW. Cyclical edema. Am J Med 1957;23:507-9.

32. Padua L, Aprile I, Caliandro P, Carboni T, Meloni A, Massi S, et al. Symptoms and neurophysiological picture of carpal tunnel syndrome in pregnancy. Clin Neurophysiol
2001;112:1946-51

33. Keir PJ, Rempel DM. Pathomechanics of peripheral nerve loading. Evidence in carpal tunnel syndrome. J Hand Ther 2005;18:259-69.

34. Pourmemari MH, Heliövaara M, Viikari-Juntura E, Shiri R. Carpal tunnel release: Lifetime prevalence, annual incidence, and risk factors. Muscle Nerve 2018;58:497-502. 